\title{
Mechanisms for pattern formation leading to axis formation and lineage allocation in mammals: a marsupial perspective
}

\author{
Lynne Selwood
}

Department of Zoology, University of Melbourne, Royal Parade, 3010 Vic, Australia

\begin{abstract}
Developing patterns in early embryogenesis are analysed in conceptuses from several families, including Dasyuridae, Phalangeridae, Macropodidae and Didelphidae, in which cleavage has been examined in some detail. Features common to cleavage and blastocyst formation, and in some cases to hypoblast formation, are used to develop an outline of possible mechanisms leading to axis formation and lineage allocation. Relevant features that have been described only in some species are also included. It is suggested that certain features of marsupial cleavage establish patterns in the developing blastocyst epithelia, pluriblast, trophoblast and hypoblast that contribute to axis formation and lineage allocation. All marsupials examined had a polarized oocyte or conceptus, the polarity of which was related to the conceptus embryonic-abembryonic axis and, eventually, the conceptus dorsal-ventral axis and the formation of the pluriblast (future embryo) and trophoblast. The embryonic dorsal-ventral and anterior-posterior axes were established after the allocation of hypoblast and epiblast. Mechanisms that appear to result in patterning of the developing epithelia leading to axis formation and lineage allocation are discussed, and include sperm entry point, gravity, conceptus polarity, differentials in cell-zona, cell-cell and cell-type (boundary effects) contacts, cell division order during cleavage and signals external to the conceptus. A model of the patterning effects is included. The applicability of these mechanisms to other amniotes, including eutherian mammals, is also examined.
\end{abstract}

Because of the simplified nature of blastocyst formation, study of marsupial development has the potential to give insights into developmental mechanisms and evolution of the mammalian embryo, especially with respect to axis formation and lineage allocation. Marsupials do not form a morula but instead the blastomeres undergo a mesenchymeepithelial transformation during cleavage to form a unilaminar epithelium in which both the pluriblast (equivalent to the inner cell mass; ICM) and the trophoblast are superficial in position. The terminology of Johnson and Selwood (1996), which allows equivalent structures in different mammalian groups to be compared, is used in this review.

Marsupial studies may indicate some evolutionary processes because marsupial conceptuses have features in common with other amniotes. Like the conceptus of reptiles and birds the marsupial conceptus is covered by a number of egg coats. The mode of formation of the early embryonic and extra-embryonic lineages is remarkably similar to that found in reptiles and birds, but can be readily seen because of the paucity of yolk material. Like the conceptus of eutherian mammals, the marsupial conceptus has to prepare itself to obtain nourishment from the uterine

Email: Iselwood@myriad.its.unimelb.edu.au environment by development of a placenta. This review will show how a study of early marsupial development reveals potential mechanisms for lineage allocation and axis formation.

\section{Conceptus and embryonic axes}

In amniotes, because early processes are concerned with establishing the extra-embryonic lineages and the embryo proper does not emerge until later, it is important to distinguish between axes developing with respect to the conceptus and those developing with respect to the embryo proper. The two axes are related but are not exactly the same (Fig. 1). Axis formation and lineage allocation are intimately related in all amniotes in which the separation of embryonic from extraembryonic lineages is preceded by the establishment of the conceptus embryonic-abembryonic axis (E/AbE). The first extra-embryonic lineages, trophoblast or extra-embryonic ectoderm of the yolk sac and then hypoblast (Fig. 1), have fundamental roles in conceptus nourishment and embryonic signalling. They also are manifestations of the first signs of the emerging dorsal-ventral (D-V) axis for the conceptus as a whole (Fig. 1). Within the epiblast, which gives rise to the future embryo (Johnson and Selwood, 1996), the definitive embryonic D-V and anterior-posterior (A-P) axes of the future 
Table 1. Possible mechanisms leading to axial gradients or lineage allocation in marsupials

\begin{tabular}{llc}
\hline Mechanisms & Evidence & References \\
\hline Uneven distribution of determinants & Oocyte or zygote polarity & Selwood and Hickford, 1999 (review) \\
Gravity & $\begin{array}{l}\text { Gravity-related orientation of conceptuses } \\
\text { in one species }\end{array}$ & Baggott and Moore, 1990 \\
Sperm entry point & $\begin{array}{l}\text { Identified in some species showing marked } \\
\text { oocyte polarity before fertilization }\end{array}$ & Merry et al., 1995; Selwood, unpublished \\
Cleavage patterns & Stylized and specific morphology & Selwood, 1992 \\
Intrinsic signals & Cell division order creates pattern in & Selwood and Smith, 1990 \\
Extrinsic signals & pluriblast and trophoblast & Sevelopmental arrests in vitro
\end{tabular}

organism emerges (Fig. 1). Apart from the small size, the appearance of the marsupial epiblast during gastrulation is similar to that of birds, reptiles and monotremes.

\section{Possible mechanisms leading to axial gradients or lineage allocation}

It is proposed here that axial gradients are set up during early development before the axis can be detected morphologically. The processes in marsupials that have the potential to create patterns in the developing blastocyst epithelium leading to either axial gradients or lineage allocation, or both, are discussed below (see Table 1).

\section{Conceptus axis formation and pluriblast-trophoblast allocation}

Oocyte-zygote polarity. Oocyte-zygote polarity is a feature encountered commonly in most marsupial families (Figs 1 and 2) and is expressed in the eccentric location of the nucleus or other organelles in oocytes and cleavage stage conceptuses, the polarized discharge of pale vesicles releasing an extracellular matrix (ECM) into the cleavage cavity or vesicles associated with cell-zona adhesion, the polarized nature of cell-zona adhesion, which is always localized initially to the hemisphere opposite vesicle emission (Fig. 3b) and the asymmetric mucoid coat in some species (Selwood and Hickford, 1999). All early conceptuses show polarity but do not all share the same polarized features. However, all early conceptuses that have been analysed ultrastructurally show polarity related to the distribution of vesicles at one pole and nucleus or mitochondrial-rich cytoplasm at the other (Selwood and Sathananthan, 1988; Baggott and Moore, 1990; Renfree and Lewis, 1996; Frankenberg and Selwood, 1998). A variety of anchoring devices characterize marsupial zygotes so that the mitochondrial-rich cytoplasm is retained when vesicles or the yolk mass are discharged (Breed et al., 1994, 1995; Merry et al., 1995; Frankenberg and Selwood, 1998).

Oocyte polarity may be related to polarized distribution of either direct determinants or indirect maternal determi- nants. Direct determinants are defined as determinants the presence of which results directly in axial gradients or lineage allocation. Indirect or pattern-forming determinants are determinants the presence of which creates a pattern leading eventually to axial gradients or lineage allocation. Polarity may be related to the uneven distribution of direct determinants because the conceptus E/AbE, D-V axis and the first lineage allocation into pluriblast and trophoblast appears to be related to the cytoplasmic polarity of the zygote (Fig. 1). The fact that the differences between pluriblast and trophoblast often disappear soon after the blastocyst epithelium is complete (one or two cell divisions) does not support this contention and indicates that polarity is more likely to be related to the distribution of indirect determinants to ensure that formation of the blastocyst epithelium is localized to one hemisphere. These two alternatives need to be tested experimentally. After cellzona adhesion begins (Fig. 2), polarity in trophoblast and pluriblast cells becomes related to the blastocyst epithelium, the outer surface of which is apical (Frankenberg and Selwood, 1998). This process is in contrast to that in eutherian mammals, in which only trophoblast cells are polarized.

Sperm entry point. Sperm entry point appears to occur preferentially in the hemisphere opposite the accumulation of vesicles that contribute to the yolk mass in dasyurid marsupials (Table 1 ) and within $60^{\circ}$ of the position of the first polar body (Fig. 3). This preference may occur because penetration in the hemisphere containing the vesicular material of the future yolk mass makes migration of the sperm head difficult or impossible. If the relationship between oocyte polarity, sperm entry point and the future $\mathrm{D}-\mathrm{V}$ axis is examined in dasyurids, some parallels with the relationship in the frog can be seen (Fig. 3) that indicate that the sperm entry point may be associated with formation of the conceptus D-V axis. This hypothesis needs to be tested experimentally. If the sperm entry point is demonstrated to play a role in conceptus $\mathrm{D}-\mathrm{V}$ axis formation, the movements of the cytoplasm after fertilization may be in response to a gravitational stimulus (Table 1). 


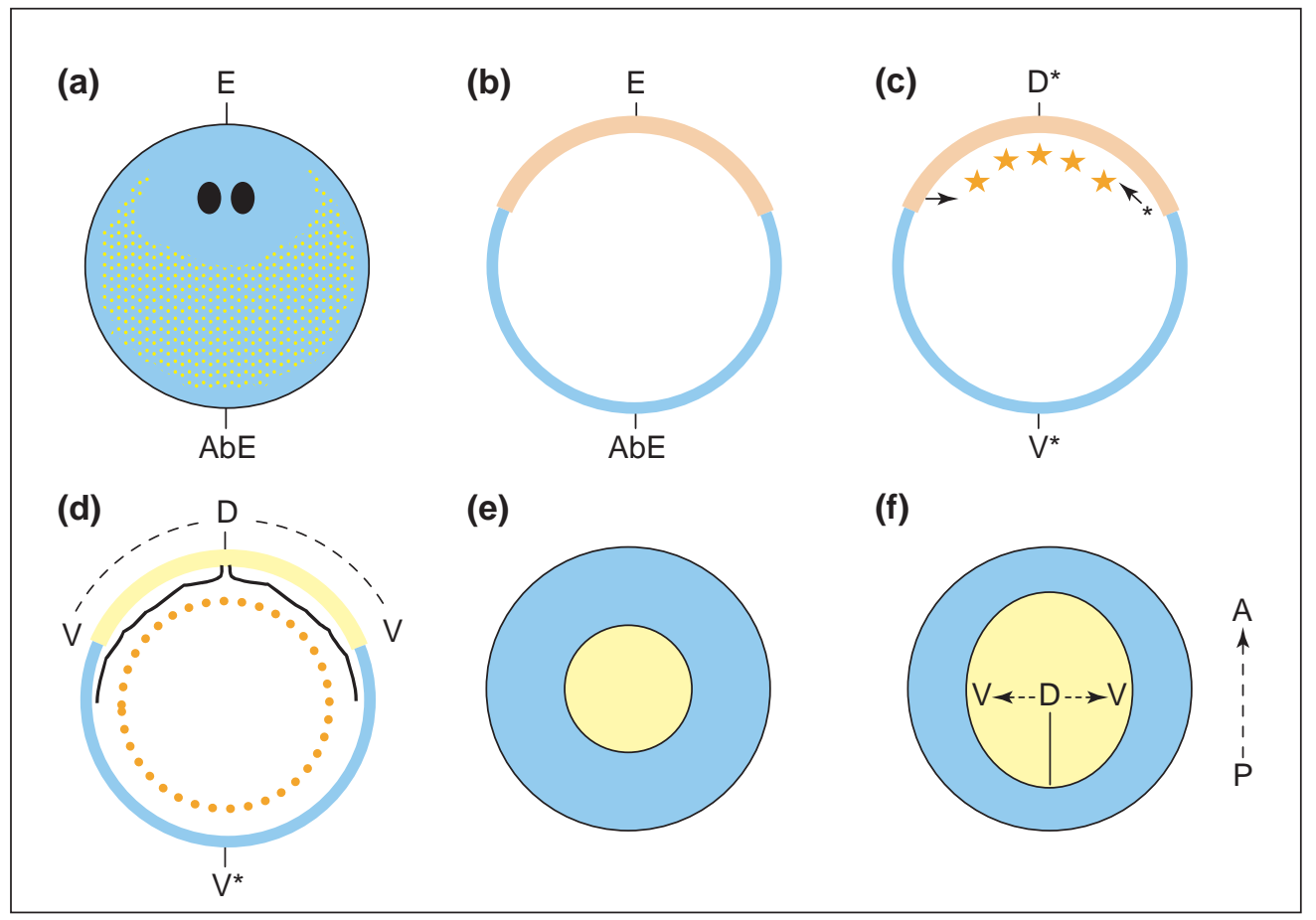

Fig. 1. Schematic representation of the developing conceptus axes and their relationship to the embryonic axes in marsupials. (a-d) Diagrammatic transverse sections. (e-f) External view of dorsal surface. (a) Zygote with polarized location of the pronuclei (closed black ovals) in vesicle-free cytoplasm (dark blue oval) and vesicle-rich cytoplasm (yellow circles). The position of the conceptus embryonic-abembryonic (E/AbE) axis is shown. (b) Unilaminar blastocyst stage in which the pluriblast (pale brown) cells are distinct from the trophoblast cells (blue) and aligned along the E/AbE axis. Pluriblast cells are more rounded and larger than the more flattened, more vesicular trophoblast cells (not shown here). (c) Early bilaminar blastocyst stage in which the allocation of hypoblast (orange stars) from the pluriblast cells marks the transition to the conceptus dorso-ventral $\left(D^{*} / V^{*}\right)$ axis. Hypoblast cells originate from the pluriblast by directed mitosis or by inward migration either from one side (*arrow) as in Antechinus stuartii or from the entire margin (arrow and *arrow) of the pluriblast as in Didelphis virginiana. (d) Trilaminar blastocyst stage in which developing mesenchymal (black) lineages between the hypoblast (orange) and overlying epiblast (yellow) initiate the transition to the embryonic D-V axis (dashed lines) which is restricted to the region of the epiblast. The $\mathrm{D}$ pole is common to both conceptus $\mathrm{D}-\mathrm{V}^{*}$ and embryonic $\mathrm{D}-\mathrm{V}$ axis. The proximal and distal trophoblast also become distinct (not shown) aligned to the conceptus $\mathrm{D}-\mathrm{V}^{*}$ axis. (e) Dorsal surface view of a bilaminar blastocyst showing the circular epiblast with no overt signs of axial gradients. (f) Dorsal surface view of an early trilaminar blastocyst in which the anterior-posterior (A-P) axis is defined initially by the increased density of epiblast cells posteriorly (not shown), the oval shape of the epiblast, then the primitive streak (black line), which extends posteriorly and also marks the most dorsal region of the embryonic $\mathrm{D}-\mathrm{V}$ axis.

Cleavage patterns. The association of conceptus polarity and the stylized and consistent cleavage patterns specific to a taxonomic group (Fig. 2) is the most powerful evidence for uneven distribution of determinants in marsupial zygotes. Cleavage patterns are holoblastic, showing accumulation of ECM into the cleavage cavity during early cleavage (Fig. 2). Only some patterns have polarized elimination of a large or several smaller membrane-bounded yolk masses and polarized discharge of ECM (Figs 2 and 3b). During cleavage, the $\mathrm{E} / \mathrm{AbE}$ axis, visible in most zygotes from the position of the nucleus (Fig. 2), becomes clearly established. The types of cleavage pattern found in marsupials, in which cleavage has been described to the 32-cell stage are shown (Fig. 2).
Each pattern shows differences in the timing of features so characteristic of marsupial cleavage. These characteristic features are: the stage of overt polarization, polarized vesicle or yolk mass elimination, cell-zona adhesion, cell-cell adhesion, allocation of pluriblast and trophoblast and the formation of a complete unilaminar blastocyst.

\section{Epiblast-hypoblast allocation and embryonic axis formation}

After establishment of the conceptus E/AbE and D-V axes and allocation of pluriblast-trophoblast lineages, the allocation of epiblast, hypoblast, proximal and distal tro- 


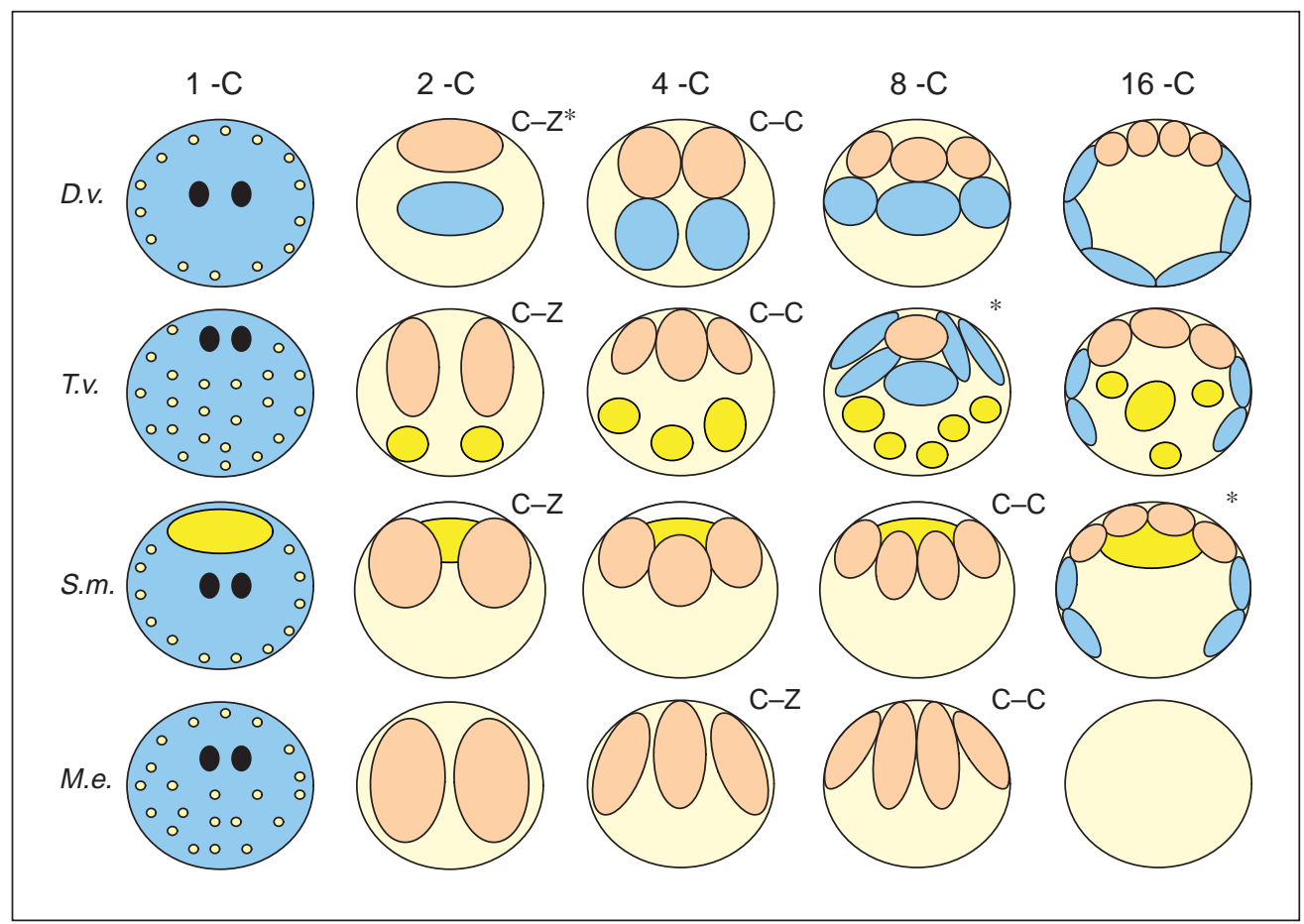

Fig. 2. Diagrams of the cleavage pattern of four marsupials, Didelphis virginiana (D.v.), Trichosurus vulpecula (T.v.) and Sminthopsis macroura (S.m.), in which all of cleavage has been described, and Macropus eugenii (M.e.) in which cleavage has been described to the eight-cell stage. The pattern in $T$. vulpecula resembles that in Monodelphis domestica and that in S. macroura resembles that in Antechinus stuartii and Dasyurus viverrinus. Representative sections through five cleavage stages (onecell to 16-cell) are shown, together with the stage at which cell-zona adhesion (C-Z), cell-cell adhesion $(\mathrm{C}-\mathrm{C})$ and formation of pluriblast and trophoblast $\left({ }^{*}\right)$ occur. Yolk masses: large closed yellow ovals and circles; pronuclei: closed black ovals; extracellular matrix: pale yellow circles in one-cell stages, and pale yellow colour in cleavage cavity in others; future pluriblast and undesignated cells: pale brown; and future trophoblast cells: blue.

phoblast and the emergence of the embryonic A-P and D-V axes occur (Fig. 1). The most likely hypothesis in marsupials is that these features emerge as a result of patterns occurring within the developing pluriblast and trophoblast epithelia. This hypothesis would fit with the capacity to regulate shown by the two most studied model amniote systems, those of the chick and the mouse. Capacity to regulate is an early feature and includes regulation for deletion or addition of cells to produce a normal blastocyst with the ICM and trophoblast in mice (Tarkowski and Wroblewska, 1967) and the rebuilding of a node in the epiblast in chicks (Yuan et al., 1995).

Marsupials do not seem to be able to replace cells destroyed in their developing blastocyst epithelia during cleavage, but once the epithelia are established, damaged cells are quickly replaced (Selwood, 1986). The lineage potency of these cells has not been tested. It is the capacity to regulate that argues most strongly against the idea that lineage allocation or axis formation is dependent on uneven distribution of direct determinants. This regulatory capacity indicates that patterns form in the developing epithelia as a result of the uneven distribution of indirect pattern-forming determinants or positional signals, or both. Positional signals may be intrinsic to the embryo (that is, within the pluriblast or epiblast) or extrinsic (that is, within the trophoblast or hypoblast or external to the conceptus). An underlying process regulating the pluriblast or trophoblast, similar to the intercalation rule suggested for regenerating systems (Bryant et al., 1977), would have to apply to maintain the patterns leading to hypoblast allocation and embryonic axis formation. The intercalation rule states that where a pattern in a field is disrupted, cells are intercalated to make up the cells with the missing values. Unlike regenerating systems in secondary fields, intercalation in the pluriblast or epiblast may be achieved without proliferation.

\section{Intrinsic signals}

The pluriblast epithelium of marsupials, unlike the ICM of mice, is unilaminar, polarized and superficial in position. This means that differential signals related to an outside 


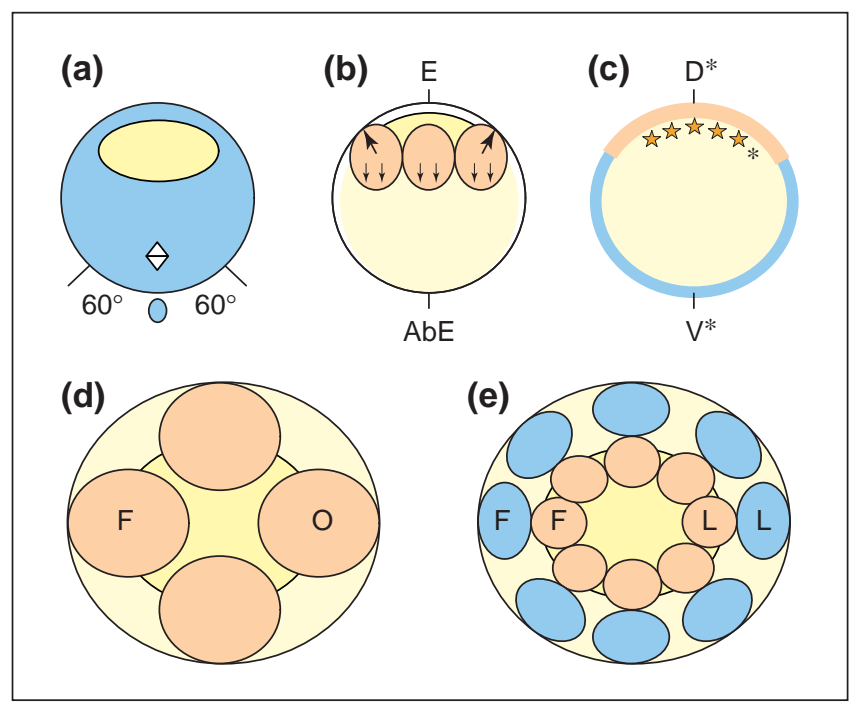

Fig. 3. Diagrams of the developing patterns in two dasyurid marsupials, Sminthopsis macroura and Antechinus stuarti. (a-c) Transverse sections showing the relationship between sperm entry point, conceptus polarity and the developing conceptus embryonicabembryonic (E/AbE) and dorsal-ventral $\left(\mathrm{D}^{*}-\mathrm{V}^{*}\right)$ axes. (d-e) Views from the dorsal side of the conceptus showing how the order of cell division can create an axis across the conceptus. (a) Polarized oocyte has a yolk mass (yellow oval at one pole with the first polar body (blue oval) and meiotic apparatus (diamond) at the other). Sperm entry point occurs within $60^{\circ}$ of the polar body. (b) Four-cell stage, showing the conceptus E/AbE axis and the polarized nature of secretions (broad arrows) leading to cell-zona adhesion at the embryonic pole and polarized secretion of ECM (small arrows) into the abembryonic hemisphere of the cleavage cavity (pale yellow). (c) Early bilaminar blastocyst in which hypoblast cells (orange stars) and the pluriblast (pale brown arc) define the conceptus dorsal $\left(D^{*}\right)$ pole and the trophoblast (blue semi-circle) defines the ventral pole $\left(\mathrm{V}^{*}\right)$. Hypoblast cells first appear on one side of the pluriblast margin $\left(^{*}\right)$. (d) Four-cell stage, in which cell division is asynchronous, showing the four blastomeres lying above the yolk mass (yellow circle). The first cell to divide (F) and the cell opposite (O) it are marked. (e) The same embryo at the 16-cell stage, showing how at the fifth division descendants (F) of the first cell to divide at the four-cell stage lie adjoining or nearby in the pluriblast ( $F$ in pale brown cell) and trophoblast ( $F$ in blue cell) are the first to divide in each lineage. The descendants of the cell opposite the first cell to divide at the four-cell stage lie on the other side of the conceptus and are the last cells to divide in each lineage of the pluriblast ( $\mathrm{L}$ in pale brown cell) and trophoblast ( $\mathrm{L}$ in blue cell). Restriction of the first and last cells to divide to opposite sides of the conceptus has the potential to create an axis across it.

(that is, adjoining the external environment) versus an inside location (that is, adjoining the blastocoele) to stimulate development of the hypoblast or primitive endoderm (Gardner, 1982) do not occur in marsupials, in which the unilaminar form means that all pluriblast cells have a similar position with respect to inside and outside. What are the potential sources of intrinsic signals causing variations to occur within the pluriblast epithelium of marsupials? Some indirect sources may be associated with uneven distribution of determinants, similar to those that occur in the vasa protein in the chick germ cell lineage (Tsunekawa et al., 2000). Several obvious candidates for intrinsic positional signals can be found during development of the epithelia, namely: differential in cell-zona contacts; differential in cell-cell contacts; boundary effects, or differential in cell type contacts; and differential in order of cell division.

Differential in cell-zona contacts. Cells developing in the centre of the developing epithelium have earlier cell-zona contacts than those at the periphery. Furthermore, the nature and mechanisms of establishment of cell-zona contacts appears to vary between central and peripheral cells in two species, Antechinus stuartii (Sathananthan et al., 1997) and Trichosurus vulpecula (Frankenberg and Selwood, 1998). This variation may be a mechanism to allocate pluriblast and trophoblast, or a consequence of the allocation. This mechanism may also separate central from peripheral cells within the pluriblast, and proximal from distal trophoblast (Fig. 2).

Differential in cell-cell contacts. In dasyurids, cells at the centre of the developing epithelium have more and earlier cell-cell contacts (Selwood and Hickford, 1999). In contrast, in the didephid Didelphys virginiana peripheral cells initially have more cell-cell contact (McCrady, 1938). This differential would re-enforce the pattern established by the differential in cell-zona contacts.

Boundary effect or differential in cell type contacts. Cells within the inner pluriblast have pluriblast-pluriblast contacts. Cells at the boundary of the pluriblast also have pluriblast-trophoblast contacts (Fig. 4a). Similar principles apply in the trophoblast. The establishment of a boundary between the pluriblast and the trophoblast has important consequences for future positional signalling between the tissues and probably contributes to the specification of ventral pluriblast-epiblast cells. With the formation of the hypoblast, additional boundary effects may be created between hypoblast and pluriblast or trophoblast (Fig. 4d).

Differential in order of cell division. In Sminthopsis macroura and Antechinus stuartii (Selwood and Smith, 1990), the descendants of the first cell to divide at the fourcell stage give rise to the first cell to divide at subsequent stages and lie on one side of the pluriblast and trophoblast (Fig. 3d,e). Descendants of the cell opposite it lie on the other side and are the last cells to divide at the eight-cell stage onwards in both pluriblast and trophoblast. This mechanism may generate a hypoblast from one side of the pluriblast and eventually an A-P axis. It is not necessarily a common mechanism because, in $D$. virginiana, the descendants of the first cell to divide at the two-cell stage 
(a)

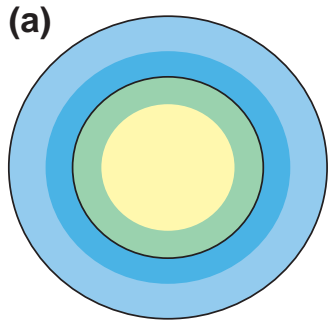

(c)

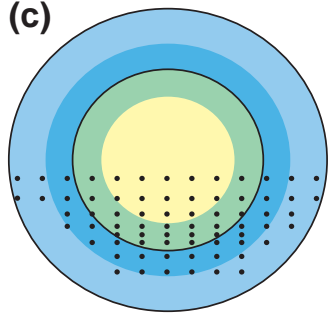

(b)

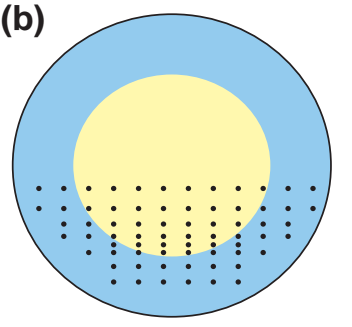

(d)

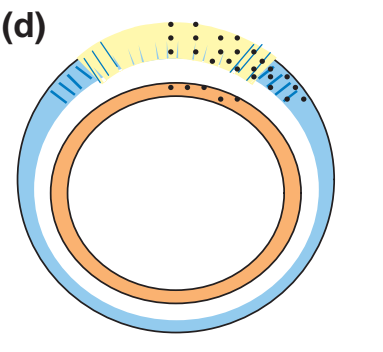

Fig. 4. Proposed patterns in conceptus epithelia in marsupials. $(a-c)$ Surface views of a blastocyst from the dorsal side showing the pluriblast-epiblast (yellow) and trophoblast (pale blue). (a) Potential boundary effects patterning (transparent blue overlay) contributed by differentials in cell-zona and cell-cell contacts so that cells at the centre of each of the pluriblast and trophoblast epithelia are different from cells in the boundaries. (b) Potential pattern contributed by differentials in cell-division order (black spots) so that one side of the conceptus is different from the other. (c) Combination of the potential patterns of (a) and (b). (d) Transverse section of a bilaminar blastocyst showing the above patterns superimposed over the epiblast (yellow arc) and trophoblast (blue) and the hypoblast (orange circle).

give rise to the upper tier of cells (McCrady, 1938) that later give rise to the future pluriblast cells (Hartman, 1916). It would be useful to see a re-evaluation of the cleavage pattern in $D$. virginiana using ultrastructural analysis.

The combined effect of these intrinsic signalling mechanisms has the potential to create the patterns (Fig. 4) that lead to axial gradients and lineage allocation in the epiblast. These patterning mechanisms, which are more readily detected in marsupials, might also apply to amniotes in general, including eutherian mammals.

\section{Extrinsic signals}

Once a pattern is formed and maintained (Fig. 4), differential gene expression across the epithelium can occur in response to a variety of positional signals either intrinsic or extrinsic, or both. A number of diverse studies indicate that marsupial embryos do respond to extrinsic signals preceding new phases in lineage allocation and axis formation. The nature of these signals is not understood. Extrinsic signals are thought to initiate and terminate embryonic diapause and other developmental arrests in marsupials (Tyndale-Biscoe and Renfree, 1987). Hypoblast formation and the development of the A-P axis follow the termination of embryonic diapause.
Relatively standard media formulations, supplemented with fetal calf serum, support culture for much of marsupial development but critical periods of development involving new lineage specification do not occur in vitro, indicating that at these times developmental progress is dependent on some unknown extrinsic signal(s). These critical stages are the proliferation of pluriblast and trophoblast after their formation, hypoblast formation and the early stages of embryonic axis formation (Selwood and Hickford, 1999).

\section{Conclusions}

Study of marsupial embryos provides a unique opportunity to examine the processes of axis formation and lineage allocation in amniotes. It may be that the processes revealed by this study are unique to marsupials, but it is much more likely that at least some of the spectrum of mechanisms used by marsupials to generate new lineages and axes will also be found in other amniotes.

This work was supported by the Australian Research Council.

\section{References}

Key references are identified by asterisks.

Baggott LM and Moore HDM (1990) Early embryonic development of the grey short-tailed opossum, Monodelphis domestica, in vivo and in vitro. Journal of Zoology (London) 222 623-639

Breed WG (1996) Egg maturation and fertilization in marsupials Reproduction, Fertility and Development 8 617-643

Breed WG, Simerly C, Navara CS, VandeBerg JL and Schatten G (1994) Microtubule configurations in oocytes, zygotes and early embryos of a marsupial, Monodelphis domestica. Developmental Biology 164 230-240

Bryant PJ, Bryant SV and French V (1977) Biological regeneration and pattern formation Scientific American 237 66-81

Frankenberg S and Selwood L (1998) An ultrastructural study of the role of an extracellular matrix during normal cleavage in a marsupial, the brushtail possum Molecular Reproduction and Development 50 420-433

*Gardner RL (1982) Investigation of cell lineage and differentiation in the extraembryonic endoderm of the mouse embryo Journal of Embryology and Experimental Morphology 68 175-198

*Hartman CG (1916) Studies in the development of the opossum Didelphys virginiana I. History of the early cleavage II. Formation of the blastocyst Journal of Morphology 27 1-83

*Johnson MH and Selwood L (1996) Nomenclature of early development in mammals Reproduction, Fertility and Development 8 759-764

McCrady E, Jr (1938) The embryology of the opossum American Anatomy Memoirs 16 1-233

Merry NE, Johnson MH, Gehring CA and Selwood L (1995) Cytoskeletal organization in the oocyte, zygote, and early cleaving embryo of the stripe-faced dunnart (Sminthopsis macroura) Molecular Reproduction and Development 41 212-224

Renfree MB and Lewis AM (1996) Cleavage in vivo and in vitro in the marsupial Macropus eugenii. Reproduction, Fertility and Development 8 725-742

Sathananthan AH, Selwood L, Douglas I and Nanayakkara K (1997) Early cleavage to formation of the unilaminar blastocyst in the marsupial Antechinus stuartii: ultrastructure Reproduction, Fertility and Development 9 201-212

Selwood L (1986) Cleavage in vitro following destruction of some blastomeres in the marsupial Antechinus stuartii (Macleay) Journal of Embryology and Experimental Morphology 92 71-84

*Selwood L (1992) Mechanisms underlying the development of pattern in marsupial embryos. In Current Topics in Developmental Biology Vol. 27 Ed. RA Pedersen pp 175-233. Academic Press, New York 
Selwood L and Hickford D (1999) Early cell lineages in marsupial embryos. In Cell Lineage and Fate Determination pp 505-519 Ed. SA Moody. Academic Press, San Diego, CA

Selwood L and Sathananthan AH (1988) Ultrastructure of early cleavage and yolk extrusion in the marsupial Antechinus stuartii. Journal of Morphology 195 327-344

Selwood L and Smith D (1990) Time-lapse analysis and normal stages of development of cleavage and blastocyst formation in the marsupials the brown antechinus and the stripe-faced dunnart Molecular Reproduction and Development 26 53-62

*Tarkowski A and Wroblewska J (1967) Development of blastomeres of mouse eggs isolated at the 4- and 8-cell stage Journal of Embryology and Experimental Morphology 18 155-180

Tsunekawa N, Naito M, Sakai Y, Nishida T and Noce T (2000) Isolation of chicken vasa homolog gene and tracing the origin of primordial germ cells Development 127 2741-50

Tyndale-Biscoe CH and Renfree MB (1987) Reproductive Physiology of Marsupials Cambridge University Press, Cambridge

Yuan S, Darnell DK and Schoenwolf GC (1995) Mesodermal patterning during avian gastrulation and neurulation: experimental induction of notochord from non-notochordal precursor cells Developmental Genetics 17 38-54 